\title{
A kinetic model of the transformation of a micropatterned amorphous precursor into a porous single crystal
}

\section{Citation}

Fratzl, Peter, Franz Dieter Fischer, Jiri Svoboda, and Joanna Aizenberg. 2010. "A Kinetic Model of the Transformation of a Micropatterned Amorphous Precursor into a Porous Single Crystal." Acta Biomaterialia 6 (3) (March): 1001-1005. doi:10.1016/j.actbio.2009.09.002.

\section{Published Version}

doi:10.1016/j.actbio.2009.09.002

\section{Permanent link}

http://nrs.harvard.edu/urn-3:HUL.InstRepos:37260714

\section{Terms of Use}

This article was downloaded from Harvard University's DASH repository, and is made available under the terms and conditions applicable to Open Access Policy Articles, as set forth at http:// nrs.harvard.edu/urn-3:HUL.InstRepos:dash.current.terms-of-use\#OAP

\section{Share Your Story}

The Harvard community has made this article openly available.

Please share how this access benefits you. Submit a story.

\section{Accessibility}




\title{
A kinetic model of the transformation of a micropatterned
}

\section{amorphous precursor into a porous single crystal}

\author{
Peter Fratzl $\left.\right|^{1 *}$, Franz Dieter Fischer², Jiři Svoboda ${ }^{3}$, Joanna Aizenberg ${ }^{4}$ \\ ${ }^{1}$ Max Planck Institute of Colloids and Interfaces, Department of Biomaterials \\ Research Campus Golm, 14424 Potsdam, Germany, fratzl@mpikg-golm.mpg.de \\ 2 Institute of Mechanics, Montanuniversität Leoben, \\ Franz-Josef-Strasse 18, A-8700 Leoben, Austria, mechanik@unileoben.ac.at \\ ${ }^{3}$ Institute of Physics of Materials, Academy of Sciences of the Czech Republic, \\ Žižkova 22, CZ-616 62, Brno, Czech Republic, svobj@ipm.cz \\ ${ }^{4}$ School of Engineering and Applied Sciences, Harvard University, \\ Cambridge, MA 02139, USA, marina@seas.harvard.edu
}

Keywords: Amorphous calcium carbonate, Biomineralisation, Crystallisation, Nucleation and growth, Phase transformation

\begin{abstract}
Biogenic single crystals with complex shapes are believed to be generated by the crystallisation of an amorphous precursor. Recent biomimetic experiments on the crystallisation of calcite via amorphous-to-crystalline transition point to the fact that the transformation kinetics may be controlled by the micropattern and the macroscopic shape of the amorphous precursor phase. Here we analyse a simple kinetic model, based on thermodynamic considerations, showing that the presence of cavities in the micropatterned precursor phase might interfere with the transformation process and control its kinetics. The size of the cavities couples to the total surface energy and, hence, to crystal nucleation and growth, while the spacing of the cavities, as compared to the typical diffusion path, controls the possible nucleation of competing crystals.
\end{abstract}

* corresponding author: E-mail: fratzl@mpikg.mpg.de

Tel.: ++49/331/567 9401 - Fax: +49-331-5679402 


\section{Introduction}

Many biological crystals are grown by the transformation of amorphous precursor phases [1-8]. For example, it was shown that amorphous calcium carbonate (ACC) transforms into calcite or aragonite in the skeletons of echinoderms and molluscs or that amorphous iron oxides transform into magnetite in chiton teeth. The advantage of this type of processing is that it apparently allows the growth of single crystals with very complex shapes $[9,10]$. It has also been argued that amorphous precursor phases modify substantially nucleation process as several subcritical nuclei may coexist, survive and eventually coalesce within a single amorphous precursor particle [11-17]. Recently, it has become possible to stabilize amorphous calcium carbonate as a precursor of calcite crystals in laboratory conditions and to initiate controlled crystallization of large single crystals [17-21].

One of the challenges in understanding the kinetics of this transformation is to rationalize the effect of the volume change between ACC and calcite, which is much denser. Hence, crystallisation of ACC is not possible without a considerable mass transport at the nano- and micrometer levels. It is quite surprising that the formation of a large single crystal is actually possible under these conditions. One would suspect that the negative volume jump from ACC to calcite would disrupt the crystallization front and the remaining ACC pool, making the growth of a single crystal difficult. Indeed, the formation of polycrystalline calcite is observed when a large plate-like ACC template transforms, even starting from a single artificial nucleation site.[18] However, when the ACC-template is pre-structured by a periodic array of holes in the plate-like template, a large single crystal may grow under similar conditions [18, 22]. As shown in Fig. 1, large single crystals with periodic arrays of holes occur in natural systems and can be grown in the laboratory. The size and the spacing of these holes seem to play a major role in the kinetics of the crystallisation process, since a single crystal is formed only when the spacing of the holes is sufficiently small. The size of the holes was observed to increase during crystallisation, obviously to compensate for the volume decrease from ACC to calcite. All these experimental observations suggested an important role of geometric constraints in the transformation process from an amorphous precursor to a single crystal, but the physical nature of this influence is still uncertain.

\section{Problem Formulation}

In this paper, we carry out theoretical investigations of the influence of geometric constraints on a phase transformation process with a considerable volume jump. The hypothesis that, perhaps, cavities in the precursor may reduce the mechanical stresses arising from the volume change [18] is not likely to be true, because the presence of holes leads to stress concentration and increases the risk of failure initiation, rather than preventing the disruption between the parent ACC phase and the crystal [23]. To avoid this, the parent phase has to be sufficiently ductile to allow for accommodation of the deformation without stress concentrations. The latter condition is fulfilled for the water-rich ACC phase, which is quite soft and deformable compared to calcite. Moreover, recent experiments of controlled crystallization of ACC show that, indeed, ACC rearranges considerably around growing crystals to form halos [19].

Here we analyse the possible influence of holes on the kinetics of the transformation of ACC to calcite, within a simple theoretical model. We find that the 
presence of cavities in the micron range can inhibit calcite nucleation and promote the growth of a single crystal from a sufficiently large artificial nucleus. We show that the time required for the crystal surface to bridge the distance a between two holes, scales as $a^{2}$, which needs to be compared to the time for nucleation of a competing crystal. Hence, a single-domain crystal will only form when the distance between holes is small enough to prevent secondary nucleation.

The model studied is (quasi-) two-dimensional, consisting of a planar, perforated layer (with a large thickness $d$ ) of ACC with the starting configuration shown in Fig. 2. The ACC layer contains a periodic array of circular holes with radius $\rho_{w}$ and the initial radius $\rho_{w_{0}}$, distributed in a square lattice with the unit cell dimension of $a$. During a diffusive transformation process the phase ACC transforms into crystalline calcite (CC), and liquid water (W). The molar fractions, molar volumes and molar Gibbs energies of individual phases are listed in Table 1.

\section{Problem Solution}

We assume that at the beginning of the phase transformation, a calcite nucleus grows to an initially very small circle around the centre with radius $\rho_{c}$, while the holes increase their radius from $\rho_{w 0}$ to $\rho_{w}$, in order to compensate for the decrease of the volume of calcium carbonate during the transformation from the ACC to the calcite phase. We postulate that the accommodation of the transformation strain, arising from significant shrinking, can be realized by the viscous behaviour of ACC. Without this property of the ACC, transformation stresses would accumulate at the interface between calcite and ACC, which must lead either to a stopping of the transformation or to a fracture of the specimen.

When the calcite crystal grows from 0 to $\rho_{c}$ and the holes grow from $\rho_{w 0}$ to $\rho_{w}$, we can write two conservation relations - one for the calcite and one for the water.

- The first one expresses the conservation of calcium ions, with $x$ being the molar fraction of water in ACC:

$$
\frac{M_{C}}{d}=\frac{\pi \rho_{C}^{2}}{\Omega_{C}}=(1-x) \frac{M_{A}}{d}=(1-x) \frac{\pi \rho_{C}^{2}+\pi \rho_{W}^{2}-\pi \rho_{W 0}^{2}}{\Omega_{A}},
$$

where it is assumed that $M_{A}$ mols of the amorphous phase is being transformed into $M_{c}$ mols of calcite and that the layer thickness $d$ remains roughly unchanged during the crystallization (that is, the mass redistribution is supposed to mainly occur within the plane of the layer and not in the third dimension). This equation can be transformed to

$$
\frac{\rho_{W}^{2}-\rho_{W 0}^{2}}{\rho_{C}^{2}}=\frac{\Omega_{A}}{(1-x) \Omega_{C}}-1 \equiv \alpha,
$$

where the parameter $\alpha$ describes the relative volume decrease when a given amount of calcium atoms transforms from ACC to calcite. The quantities $\Omega_{A}$ and $\Omega_{C}$ are the molar volumes of ACC and calcite (see Table 1).

- The second conservation relation defines the total number $M_{w}$ of mols of water which had to leave the amorphous phase during the transformation process:

$$
\frac{M_{w}}{d}=x \frac{M_{A}}{d}=\frac{x}{1-x} \frac{\pi \rho_{C}^{2}}{\Omega_{C}} .
$$


The change $\Delta G$ in the total Gibbs free energy $G$ (within the unit cell in Fig. 2) due to the nucleation of a calcite crystal of size $\rho_{c}$ is given by a sum of the contributions from the bulk phases and from their respective interfaces as

$$
\Delta G=-M_{A} g_{A}+M_{C} g_{C}+M_{w} g_{w}+2 \pi d\left(\rho_{C} \gamma_{C}+\left(\rho_{w}-\rho_{w 0}\right) \gamma_{w}\right),
$$

(for definitions see Table 1). Using relations (1) to (3), we can eliminate $\rho_{w}$ in the expression for $\Delta \mathrm{G}$, and obtain:

$$
\begin{gathered}
\Delta G / d=-\pi \rho_{C}^{2} \hat{g}+2 \pi\left(\gamma_{C} \rho_{C}+\gamma_{w}\left(\sqrt{\rho_{w_{0}}^{2}+\alpha \rho_{C}^{2}}-\rho_{w 0}\right)\right), \\
\hat{g}=\frac{g_{A}-(1-x) g_{C}-x g_{w}}{(1-x) \Omega_{C}} \geq 0 .
\end{gathered}
$$

The expression $\hat{g}$ corresponds to the decrease in molar Gibbs energy during the transformation. The energy difference $\Delta G / d$ must be positive to allow for crystallization. This expression neglects the change of the ACC-water interface into a calcite water interface on the upper and lower sides of the slab. Taking this contribution into account, $\hat{g}$ would have to be replaced by $\hat{g}+2\left(\gamma_{w}-\gamma_{w}^{\prime}\right) / d$, where $\gamma_{w}^{\prime}$ is the surface energy calcite-water. We estimate $\hat{g}$ to be in the order of $1.310^{8}$ $\mathrm{J} / \mathrm{m}^{3}$ [24]. This value is obtained by using the following thermodynamic values reported for calcite and the hydrated calcium carbonate mineral monohydrocalcite (as we do not have good thermodynamic data for the hydrated ACC): $g_{A} /(1-x)=-1537 \mathrm{~kJ} / \mathrm{mol}$ and $x=1 / 2$ for monohydrocalcite, $g_{C}=-1235 \mathrm{~kJ} / \mathrm{mol}$ for calcite, $g_{W}=-307 \mathrm{~kJ} / \mathrm{mol}$ for water at $25^{\circ} \mathrm{C}$ [24] and $\Omega_{C}=3.6910^{-5} \mathrm{~m}^{3} / \mathrm{mol}$. (Note that taking ikaite instead of monohydrocalcite for this estimate, one would find $\hat{g}$ to be in the order of $310^{8} \mathrm{~J} / \mathrm{m}^{3}$ ). We do not have good data for $\gamma_{w}$, but the calcitewater interface has been reported to be in the order of $\gamma_{w}^{\prime}=0.1 \mathrm{~J} / \mathrm{m}^{2}$ [25]. Taking $\gamma_{w}-\gamma_{w}^{\prime}$ to be in the same order, then $2\left(\gamma_{w}-\gamma_{w}^{\prime}\right) / d$ will be much smaller than $\hat{g}$, for layer thicknesses in the micron or millimetre range. It is, therefore, sufficient to consider $\hat{g}$ in equation (5).

The analysis of this expression (5) also suggests that the presence of the holes adds surface energy (which depends differently on $\rho_{c}$ than the $\hat{g}$ term) to the system and, therefore, reduces the growth rate of a nucleus. The calcite nucleus will grow if its radius is larger than the value of $\rho_{c}$ that minimizes $\Delta G$. To get a simple understanding of eq. (5), we first assume that the interface energy between calcite and ACC is much lower than that between ACC and water and, if we set $\gamma_{C}$ to zero in the derivative of eq. (5) with respect to $\rho_{C}$, one finds that the calcite nucleus can grow, if

$$
\rho_{w 0}^{2}+\alpha \rho_{C}^{2} \geq\left(\alpha \gamma_{w} / \hat{g}\right)^{2}=\tilde{\rho}^{2} .
$$

As a consequence, an initial hole that is large enough (the critical value being $\rho_{\mathrm{w}_{0}}>\tilde{\rho}$ ) does NOT hinder the growth of the calcite nucleus. In order to get a first approximation for $\rho_{\mathrm{w}_{0}}$, we take $\gamma_{w} \approx 0.1 \mathrm{~J} / \mathrm{m}^{2}$ and $\hat{g} \approx 1.310^{8} \mathrm{~J} / \mathrm{m}^{3}$. With a volume increase $\alpha$ being on the order of 1 ( $\alpha=0.33$ for monohydrocalcite and $\alpha=2$ for ikaite) the order of magnitude for $\tilde{\rho}$ is then about one nanometer. This is quite small 
and, under such circumstances, an initial hole will not change the growth behaviour of the calcite nucleus. The situation would, however, be completely different in a setting where the ACC is stabilized (e.g. by some additive) so that the driving force for crystallization, $\hat{g}$, would be reduced to, say, $10^{6} \mathrm{~J} / \mathrm{m}^{3}$ or less. Then the critical size for the initial hole would be in the order of micrometers and the growth of the calcite nucleus would be strongly influenced by the presence of the holes.

One may imagine two scenarios:

1) $\rho_{\mathrm{w}_{0}}>\tilde{\rho}$ : Under these conditions, the initial holes in the ACC layer do not essentially influence the growth of the calcite nucleus (except for a slight reduction of the driving force $\hat{g}$ )

2) $\rho_{\mathrm{w}_{0}}<\tilde{\rho}$ : Under these conditions, the holes in the structure are hindering the growth of calcite nuclei smaller than $\sqrt{\left(\tilde{\rho}^{2}-\rho_{w 0}^{2}\right) / \alpha}$. Typically this value will be in the same order of magnitude as $\tilde{\rho}$, and this means that calcite nucleation will effectively be suppressed, if $\tilde{\rho}$ is in the order micrometers. Starting from a single (sufficiently large) nucleus, this situation might favour the growth of a single crystal during the crystallization of ACC.

To obtain some information about the transformation kinetics, one need to consider that water must be transported by diffusion in ACC from the site where calcite transformation occurs to the interfaces. This is sketched in Fig. 3. Depending on the distance of the transformation front to the nearest hole in relation to the slab thickness, the water flux will be predominantly to the hole or to the surface above and below. Viscous flow then drives the calcium carbonate in the opposite direction. Typically, the calcite crystal grows until all the calcium carbonate present in the near neighbourhood has been consumed. For isolated nuclei, this apparently leads to circular regions around each calcite crystal where ACC has been depleted [19]. In particular, if there are no holes in the structure, exchange is only possible with the upper and lower surface. However, if the $a / d$ ratio is small enough, diffusion will be mostly in-plane transporting water from the transformation front into the hole. This is the situation considered in our model.

Assuming a simplified field of diffusive flux of water consisting of two overlapping radial fields (see Fig. 2) and that there is no water in the calcite phase and no water at the hole interface (water is getting out of the solid phase at the hole), we can derive simple solutions for the fluxes in terms of $1 /$ distance; specifically, see e.g. [26] Sect 3:

$$
j_{C}=\dot{\rho}_{C} \frac{x}{\Omega_{A}} \frac{\rho_{C}}{r_{C}} \text { and } j_{W}=\dot{\rho}_{W} \frac{x}{\Omega_{A}} \frac{\rho_{W}}{r_{W}} .
$$

With these fluxes, we can calculate the total energy dissipation from diffusion (according to [27], section 3, and App. B there) as

$$
Q / d=\int_{\rho_{c}}^{a / 2} \frac{j_{C}^{2}}{A_{C}} 2 \pi r_{C} d r_{C}+\int_{\rho_{W}}^{a / 2} \frac{j_{W}^{2}}{A_{W}} 2 \pi r_{W} d r_{W}, \text { with } A_{C}=A_{W}=\frac{x D_{W}}{\Omega_{A} R_{g} T},
$$

where $R_{\mathrm{g}}$ is the gas constant, $T$ is the temperature, $D \mathrm{w}$ is the diffusion coefficient of water in ACC and the quantities $A_{c}, A_{w}$ are the "bulk" mobilities of water. Using eq. (2) in rate form, $\rho_{w} \dot{\rho}_{w}=\alpha \rho_{c} \dot{\rho}_{C}$, the abbreviations $y=2 \rho_{c} / a, \beta=2 \rho_{w 0} / a$, and $a$ renormalized time $\tau=D_{w} t / a^{2}$, we get: 


$$
Q / d=-\frac{\pi x R_{g} T D_{w}}{16 \Omega_{A}} \dot{y}^{2} y^{2}\left(2 \ln y+\alpha^{2} \ln \left(\beta^{2}+\alpha y^{2}\right)\right),
$$

where $\dot{y}=d y / d \tau$. The kinetics of a system follows from Onsager's principle of maximizing the dissipation $Q$ with the boundary condition $Q=-\dot{G}=-\Delta \dot{G}$ (for details on this principle see [28] and for applications on diffusion problems see [27] and later [29]). In the case at hand, we have for a fixed value of $\alpha$ only one internal variable describing the system, namely $\rho_{\mathrm{C}}$ and its rate $\dot{\rho}_{\mathrm{C}}$, which can immediately be found by equating $-\dot{G}$ from eq. (5), and $Q$ from eq. (9). Neglecting the contribution from the surfaces at sufficiently large times, we arrive to: $-\dot{G} / d=\pi D_{w} y \dot{y} \hat{g} / 2$. Equating this with (9), leads to a differential equation in $y$ which can be analytically integrated to give:

$$
K \tau=\left(1+\alpha^{2}\right) y^{2}+\alpha \beta^{2} \ln \beta^{2}-\left[y^{2} \ln y^{2}+\alpha\left(\beta^{2}+\alpha y^{2}\right) \ln \left(\beta^{2}+\alpha y^{2}\right)\right],
$$

where $K=16 \Omega_{A} \hat{g} /\left(x R_{g} T\right)$ is a dimensionless constant. Fig. 4 shows graphs of the function $y(\tau)$ for various values of the parameters $\alpha$ and $\beta$.

It is apparent from the growth kinetics shown in Fig. 4 that, at fixed parameters $\alpha$ and $\beta$, the time for the crystal to grow towards the next hole scales with $a^{2}$ and is not linear in a. As a consequence, we expect that, if the distance between holes becomes too large, the time for the single crystal to grow becomes large compared to the time needed for the nucleation of other crystals. If the first crystal has grown to the neighbouring hole, the model can be applied again with the nucleus now being at the advancing crystal front. Therefore, the time for the crystal to grow over many holes will scale linearly with the number of holes (and not quadratically). The dependence on the intial size of the holes (that is, on the dimensionless parameter $\beta$ ) is weak (see Fig. 4), as long as it is large enough to overcome the surface tension (see the discussion after eq. 6). The dependence on the volume jump $\alpha$ from ACC to calcite is, however, important with a considerable retardation of the crystallisation when $\alpha$ gets larger.

\section{Discussion and Conclusion}

Our theoretical analysis of the transformation process of the micropatterned ACC to calcite shows that the existence of cavities in a slab-like amorphous precursor has several profound consequences for the crystallization kinetics:

1) The cavities may act as a sink for water to compensate for the volume change during the crystallization of ACC into calcite. This requires, however, that the thickness $d$ of the precursor slab should not be much smaller than the spacing between cavities, so that $a / d$ should be in the order of unity or less.

2) In addition, small cavities hinder the growth of calcite nuclei. In particular, if the driving force for crystallization is small enough and the size of the cavities are below a critical threshold, the nucleation of calcite is suppressed, and only one large nucleus may grow (eventually into a single crystal).

3) The transformation time scales with $a^{2}$ and the calcite crystal nucleus grows to a given size roughly as the square-root of time. This means that for large values of $a$, the nucleation rate of competing crystals from the same precursor might also prevent the growth of a single crystal, even if condition 2 is fulfilled. 
These mechanistic considerations are not only in agreement with the existing experimental data [18], but they also add a higher level of understanding of the crystallization process. Indeed, for a slab thickness $d$ of ten micrometers and a spacing a between holes in the same order, the formation of a single crystal from a single nucleus has been found experimentally (Fig. 1). When the spacing a was increased to 100 microns, however, polycrystalline calcite appeared [18] (in agreement with the fact that $a / d>>1$ ). The fact that the transformation starts and progresses from a single nucleation site indicates that heterogeneous nucleation is rather low (except at the artificial nucleus) in this system. This fact justifies the current modelling approach which would not be valid in a situation where heterogeneous nucleation (e.g. at walls or surfaces) dominates. In particular, the above analysis confirms the importance of geometric constraints on the transformation kinetics and enables a rational design of the crystallization environment and space. It provides the absolute value of the cavity spacing $a$, of its ratio to the slab thickness $d$ and of the cavity size required to optimize the probability for the growth of a large single crystal from an amorphous precursor. Using these parameters, one can generate artificial crystals of arbitrary shapes similar to the convoluted micropatterns of their biogenic single-crystalline counterparts. Finally, one should be quite careful, however, in extrapolating these considerations to single crystal formation in biological organisms where the amorphous precursor phase is often anhydrous [8]. In such situations, water diffusion can not be the controlling mechanism, but it is not unlikely that the diffusion of other ions or molecules stabilizing the precursor phase [30] could play a similar role.

\section{References}

[1] Towe KM, Lowenstam HA. Ultrastructure and Development of Iron Mineralization in Radular Teeth of Cryptochiton Stelleri (Mollusca). J Ultrastruct Res 1967;17,1-13.

[2] Beniash E, Aizenberg J, Addadi L, Weiner S. Amorphous calcium carbonate transforms into calcite during sea urchin larval spicule growth. Proc Roy Soc London B 1997;264,461-465.

[3] Addadi L, Raz S, Weiner S. Taking advantage of disorder: Amorphous calcium carbonate and its roles in biomineralization. Advanced Materials 2003;15,959-970.

[4] Aizenberg J, Weiner S, Addadi L. Coexistence of amorphous and crystalline calcium carbonate in skeletal tissues. Conn Tissue Res 2003;44,20-25.

[5] Weiner S, Sagi I, Addadi L. Choosing the crystallization path less traveled. Science 2005;309,1027-1028.

[6] Ma Y, Weiner S, Addadi L. Mineral deposition and crystal growth in the continuously forming teeth of sea urchins. Adv Func Mater 2007;17,2693-2700.

[7] Gago-Duport L, Briones MJI, Rodriguez JB, Covelo B. Amorphous calcium carbonate biomineralization in the earthworm's calciferous gland: Pathways to the formation of crystalline phases. J Struct Biol 2008;162,422-435.

[8] Politi Y, Metzler RA, Abrecht M, Gilbert B, Wilt FH, Sagi I, Addadi L, Weiner S, Gilbert P. Transformation mechanism of amorphous calcium carbonate into calcite in the sea urchin larval spicule. Proceedings of the National Academy of Sciences of the United States of America 2008;105,17362-17366.

[9] Aizenberg J, Tkachenko A, Weiner S, Addadi L, Hendler G. Calcitic microlenses as part of the photoreceptor system in brittlestars. Nature 2001;412,819-822.

[10] Colfen $\mathrm{H}$. Single crystals with complex form via amorphous precursors. Angew Chem Int Ed 2008;47,2351-2353. 
[11] Zhang TH, Liu XY. How does a transient amorphous precursor template crystallization. J Am Chem Soc 2007;129,13520-13526.

[12] Rieger J, Frechen T, Cox G, Heckmann W, Schmidt C, Thieme J. Precursor structures in the crystallization/precipitation processes of $\mathrm{CaCO} 3$ and control of particle formation by polyelectrolytes. Farday Disc 2007;136,265-277.

[13] Neumann M, Epple M. Monohydrocalcite and Its Relationship to Hydrated Amorphous Calcium Carbonate in Biominerals. Eur J Inorg Chem 2007,1953-1957.

[14] Nebel H, Neumann M, Mayer C, Epple M. On the Structure of Amorphous Calcium Carbonates. A Detailed Study by Solid-State NMR Spectroscopy. Inorg Chem 2008;47,78747879.

[15] Colfen $\mathrm{H}$, Mann S. Higher-order organization by mesoscale self-assembly and transformation of hybrid nanostructures. Angew Chem Int Ed 2003;42,2350-2365.

[16] Gebauer D, Volkel A, Colfen H. Stable Prenucleation Calcium Carbonate Clusters. Science 2008;322,1819-1822.

[17] Pouget EM, Bomans PHH, Goos JACM, Frederik PM, de With G, Sommerdijk NAJM. The Initial Stages of Template-Controlled CaCO3 Formation Revealed by Cryo-TEM. Science 2009;323,1555-1458.

[18] Aizenberg J, Muller DA, Grazul JL, Hamann DR. Direct fabrication of large micropatterned single crystals. Science 2003;299,1205-1208.

[19] Han TYJ, Aizenberg J. Calcium carbonate storage in amorphous form and its template-induced crystallization. Cham Mater 2008;20,1064-1068.

[20] Kim YY, Douglas EP, Gower LB. Patterning inorganic (CaCO3) thin films via a polymer-induced liquid-precursor process. Langmuir 2007;23,4862-4870.

[21] Lam RSK, Charnock JM, Lennie A, Meldrum FC. Synthesis-dependant structural variations in amorphous calcium carbonate. Cryst Eng Comm 2007;9,1226-1236.

[22] Meldrum FC, Ludwigs S. Template-directed control of crystal morphologies. Macromol Biosci 2007;7,152-162.

[23] Kienzler R, Fischer FD, Fratzl P. On energy changes due to the formation of a circular hole in an elastic plate. Arch Appl Mech 2006;76,681-697.

[24] Königsberger E, Königsberger LC, Gamsjäger H. Low-temperature thermodynamic model for the system Na2CO3-MgCO3-CaCO3-H2O. Geochim Cosmochim Acta 1999;63,3105-3119.

[25] Aquilano D, Calleri M, Natoli E, Rubbo M, Sgualdino G. The $\{104\}$ cleavage rhombohedron of calcite: theoretical equilibrium properties. Mater Chem Phys 2000;66,159163.

[26] Fischer FD, Simha NK. Influence of material flux on the jump relations at a singular interface in a multicomponent solid. Acta Mech 2004;171,213-223.

[27] Svoboda J, Fischer FD, Fratzl P, Kroupa A. Diffusion in multi-component systems with no or dense sources and sinks for vacancies. Acta Mater 2002;50,1369-1381.

[28] Svoboda J, Turek I, Fischer FD. Application of the thermodynamic extremal principle to modeling of thermodynamic processes in material sciences. Philos Mag 2005;85,36993707.

[29] Svoboda J, Fischer FD, Fratzl P. Diffusion and creep in multi-component alloys with non-ideal sources and sinks for vacancies. Acta Mater 2006;54,3043-3053.

[30] Al-Sawalmih A, Li C, Siegel S, Fratzl P, Paris O. On the Stability of Amorphous Minerals in Lobster Cuticle. Adv Mater 2009;published online 10.1002/adma.200900295 
Tables

\begin{tabular}{|c|c|c|c|c|c|}
\hline & \multicolumn{3}{|c|}{ bulk properties } & \multicolumn{2}{|c|}{ Interface properties } \\
\hline Phase & $\begin{array}{c}\text { Molar } \\
\text { fraction } \\
\mathrm{H}_{2} \mathrm{O}\end{array}$ & $\begin{array}{c}\text { Molar } \\
\text { volume } \\
\mathrm{m}^{3} / \mathrm{mol}\end{array}$ & $\begin{array}{c}\text { Molar Gibbs } \\
\text { energy } \\
\mathrm{J} / \mathrm{mol}\end{array}$ & \multirow[t]{2}{*}{$\begin{array}{c}\text { Interface } \\
\text { energy } \\
\mathrm{J} / \mathrm{m}^{2}\end{array}$} & $\begin{array}{c}\text { Radius } \\
\mathrm{m}\end{array}$ \\
\hline Water & 1 & $\Omega_{w}$ & $g_{\mathrm{w}}$ & & \multirow[b]{2}{*}{$\rho w$} \\
\hline$\Delta \subset C$ & v & $O(x)$ & $a_{1}$ & $\gamma_{w}$ & \\
\hline & & & & $\gamma_{C}$ & \multirow[t]{2}{*}{$\rho_{c}$} \\
\hline Calcite & 0 & $\Omega_{C}$ & $g_{\mathrm{C}}$ & & \\
\hline
\end{tabular}

Table 1: Definition of thermodynamic quantities for the different phases (columns two to four) and of the interfaces between Water and ACC and between Calcite and ACC (last two columns). 


\section{Figures and Captions}
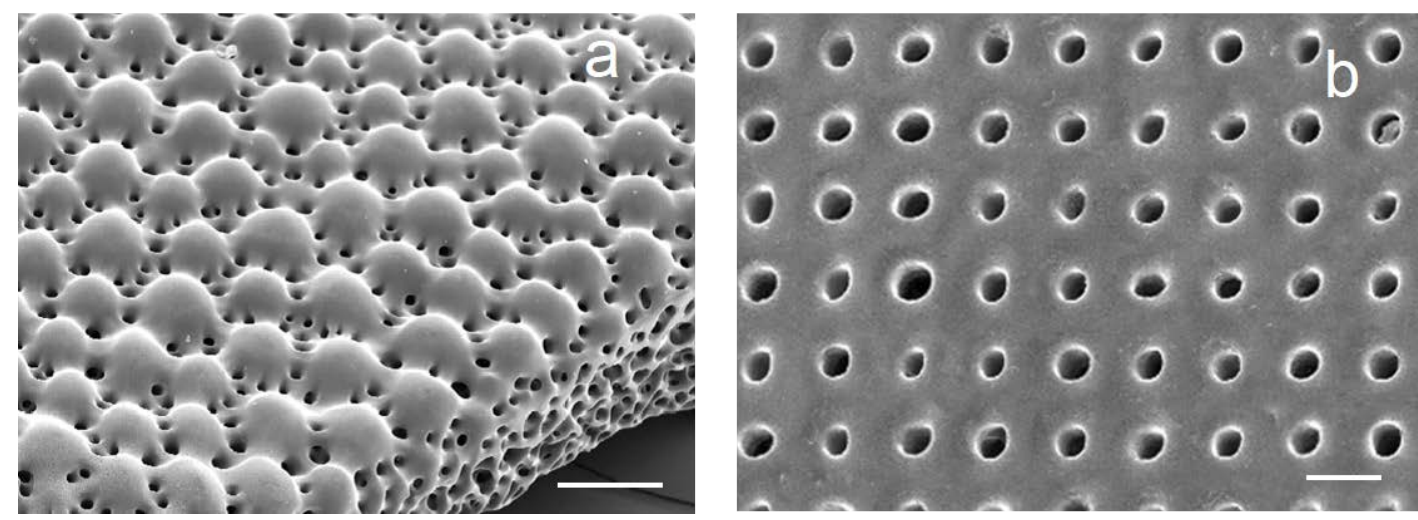

Fig. 1. (a) Scanning electron micrograph (SEM) of a part of the skeleton of a brittlestar Ophiocoma wendtii (Ophioroidea, Echinodermata). The entire structure (the mesh and the array of microlenses) is composed of a single calcite crystal used by the organism for mechanical and optical functions [9]. (b) SEM of a sample micro-patterned single calcite crystal fabricated by transformation of an amorphous precursor. The holes were pre-existing in the precursor and grew during the transformation process [18].

Bar $=100 \mu \mathrm{m}$ and $10 \mu \mathrm{m}$ in a and in $\mathrm{b}$, respectively.

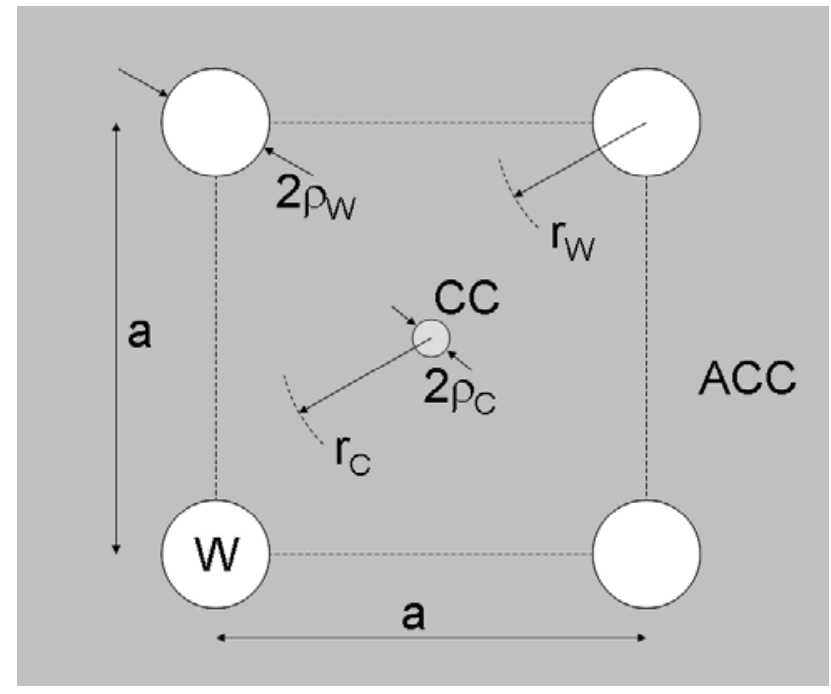

Fig. 2: Sketch of the configuration of holes (W) and the calcite nucleus (CC) in the ACC plate. The holes with radius $\rho_{w}$ (with $\rho_{w_{0}}$ being its initial value at the start of crystallization) are arranged on a square lattice with spacing a. The radius of the calcite nucleus is $\rho_{C}$., and the distance from the centre of the calcite nucleus and of the holes is $r_{c}$ and $r_{W}$, respectively. 


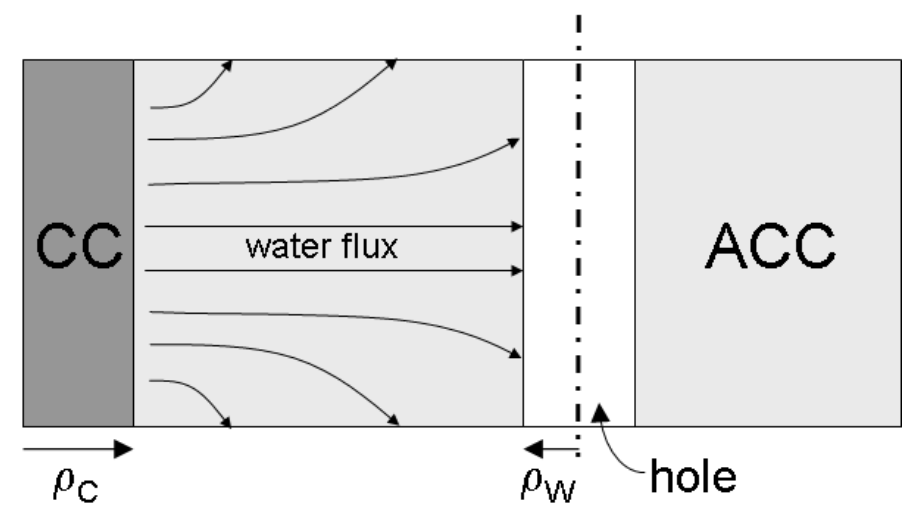

Fig. 3: Water flux from the transformation front to the specimen surface.

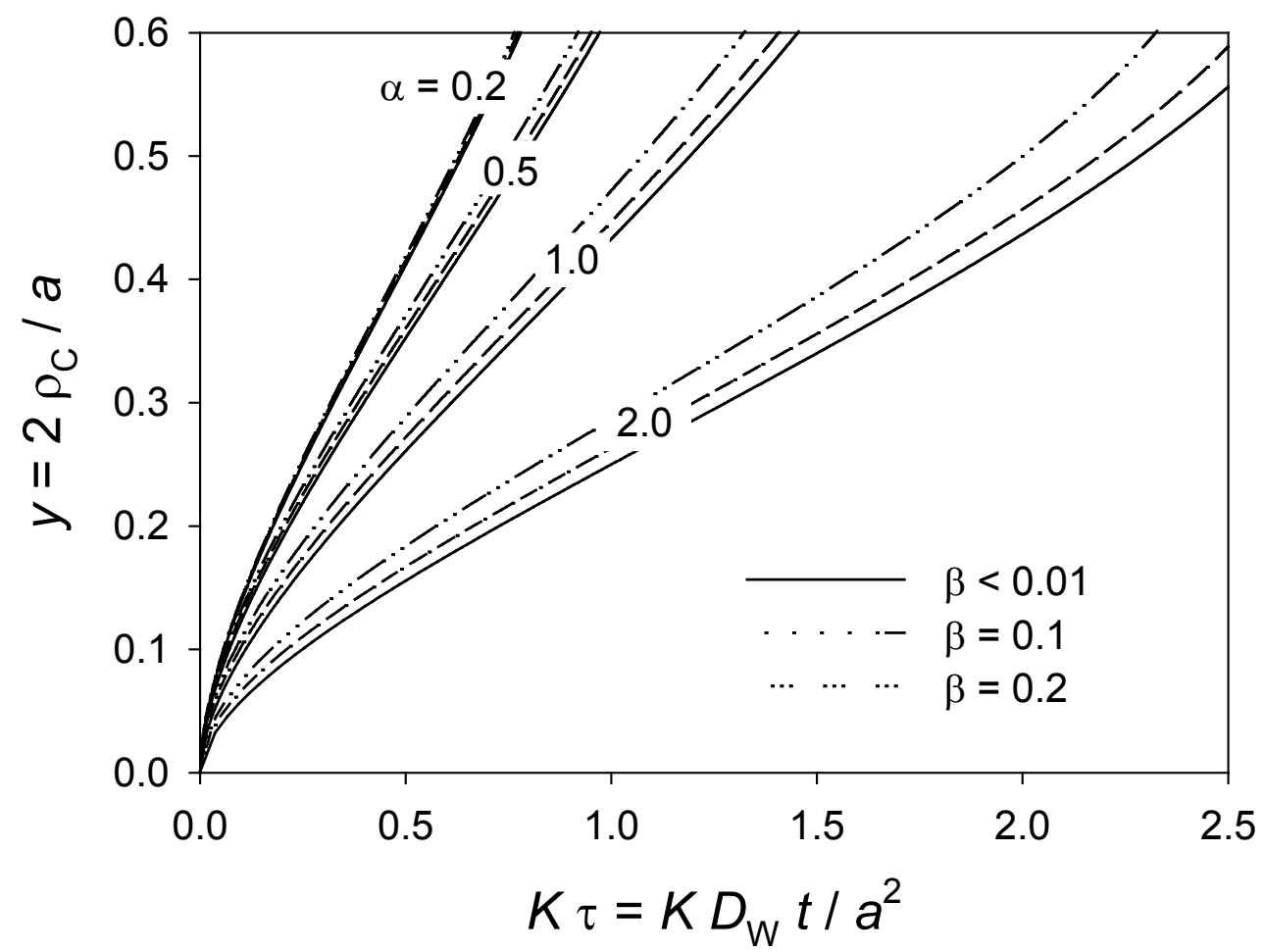

Fig. 4: Plots of the nucleus diameter normalized by the lattice spacing, $y$, as a function of normalized time $K \tau$ for several values of the relative volume jump, $\alpha$, and the initial hole diameter relative to the lattice spacing, $\beta$, according to eq. (10). 